\title{
Motor-mediated bidirectional transport along an antipolar microtubule bundle: A mathematical model
}

\author{
Congping Lin,,${ }^{1,2, *}$ Peter Ashwin, ${ }^{1}$ and Gero Steinberg ${ }^{2}$ \\ ${ }^{1}$ Mathematics Research Institute, University of Exeter, Exeter, United Kingdom, EX4 4QF \\ ${ }^{2}$ School of Biosciences, University of Exeter, Exeter, United Kingdom, EX4 4QD
}

(Received 4 August 2012; revised manuscript received 19 February 2013; published 16 May 2013)

\begin{abstract}
Long-distance bidirectional transport of organelles depends on the coordinated motion of various motor proteins on the cytoskeleton. Recent quantitative live cell imaging in the elongated hyphal cells of Ustilago maydis has demonstrated that long-range motility of motors and their endosomal cargo occurs on unipolar microtubules (MTs) near the extremities of the cell. These MTs are bundled into antipolar bundles within the central part of the cell. Dynein and kinesin-3 motors coordinate their activity to move early endosomes (EEs) in a bidirectional fashion where dynein drives motility towards MT minus ends and kinesin towards MT plus ends. Although this means that one can easily assign the drivers of bidirectional motion in the unipolar section, the bipolar orientations in the bundle mean that it is possible for either motor to drive motion in either direction. In this paper we use a multilane asymmetric simple exclusion process modeling approach to simulate and investigate phases of bidirectional motility in a minimal model of an antipolar MT bundle. In our model, EE cargos (particles) change direction on each MT with a turning rate $\Omega$ and there is switching between MTs in the bundle at the minus ends. At these ends, particles can hop between MTs with rate $q_{1}$ on passing from a unipolar to a bipolar section (the obstacle-induced switching rate) or $q_{2}$ on passing in the other direction (the end-induced switching rate). By a combination of numerical simulations and mean-field approximations, we investigate the distribution of particles along the MTs for different values of these parameters and of $\Theta$, the overall density of particles within this closed system. We find that even if $\Theta$ is low, the system can exhibit a variety of phases with shocks in the density profiles near plus and minus ends caused by queuing of particles. We discuss how the parameters influence the type of particle that dominates active transport in the bundle.
\end{abstract}

DOI: 10.1103/PhysRevE.87.052709

PACS number(s): 87.10.Mn, 87.10.Hk, 87.16.Wd

\section{INTRODUCTION}

Spatial redistribution of organelles is of central importance to all eukaryotic cells. Long-distance transport involves the activity of molecular motors (powered by hydrolysis of ATP [1]) along polymers of tubulin dimers, the so-called microtubules (MTs). Bidirectional transport along MTs involves coordination between opposing motor molecules kinesin and dynein where kinesin takes cargos towards plus ends of MTs and dynein towards minus ends [2]. Since the MTs are approximately linear, this poses the question of how bidirectional motility can occur on the same bundle (or even the same MT) without forming blockages that would give a loss of transport efficiency.

Various modeling studies have helped to illuminate the cooperative aspects of the motility of cargos along MTs (see, e.g., [3-5]). These tend to be based around extensions of the asymmetric simple exclusion process (ASEP) on a single track (see the review [6] and references therein). Extended ASEP models for bidirectional transport have explored various ways to overcome collisions between opposite-directed particles. One approach assumes that particles bind to and unbind from tracks [7-10] to allow particles to pass each other. Another approach assumes that the exclusion principle only applies to particles moving in the same direction and the presence of a motor in the opposite direction modifies the rate at which motors enter a site [11]. Alternatively, a high direction-change rate can avoid clusters due to collisions [12].

${ }^{*}$ Corresponding author: C.Lin@exeter.ac.uk
Moreover, Evans et al. introduced another possibility [13] to avoid collisions by allowing particle interchanges when they meet. Juhász introduced a two-lane ASEP [14] with opposite-directed particles moving in separated lanes, thereby avoiding collisions between opposite-directed particles. The two-lane ASEP is equivalent to a two-species ASEP in some sense as discussed in [15]. We consider a multilane model in [16] where particles can change between protofilaments to avoid collisions, using the fact that a single MT consists of 13 protofilaments [17], each of which provides a potential track for motors.

Based on recent advances in live cell imaging techniques and the use of the fungal model system Ustilago maydis, in vivo observation of dynein indicates that collision between opposite-directed motors rarely occurs $[18,19]$. This suggests that we can adapt the two-lane model in [14] to investigate the bidirectional transport of dynein motors on unipolar MTs. The adapted two-lane model together with a more sophisticated 13-lane model provided an explanation for the formation of dynein accumulation at MT plus ends [16,18,20]. The suggested function [18] of the dynein accumulation at MT plus ends is to prevent the cargo of early endosomes (EEs) falling off the MT. More recent work on U. maydis has shown that the majority of the fungal cell contains antipolar MT bundles and that unipolar MTs are restricted to the cell poles [21].

Early endosomes are the main cargo of dynein and kinesin-3 in hyphal cells $[22,23]$ and undergo long-distance bidirectional motility [24]. Interestingly, experimental evidence suggests that bidirectional long-distance motility of EEs along the bipolar MTs is mainly mediated (dominated) by kinesin-3, whereas dynein is mediating retrograde motility of EEs along 
the unipolar MT [21] and observes that EEs can travel over the entire length of the MT array. In conclusion, [21] shows that dynein and kinesin-3 cooperate and that cargo can hop between MTs within the bundle. It is also shown that during the EE transport, dynein can detach from the cargo and the EE continues motility (carried by kinesin-3) after a short pause. Moreover, short pauses of EEs before they continue the directed motility are observed at minus ends of MTs by visualizing both minus ends of MTs and EEs. These observations suggest that EEs may change MTs at MT minus ends by altering their active motor types.

Hopping between tracks has been modeled for unidirectional traffic [25-29] where a single track splits into parallel tracks. The junction between single and parallel tracks allows particles on the single track to step into either of the parallel tracks. Here we provide a model that is based on a previous ASEP model [20], but which includes a junction mechanism to describe bidirectional motility of cargo along an antipolar microtubule bundle. In our model, the lattice is composed of two MTs that are coupled at microtubule minus ends and the arrangement of the antipolar MT bundle gives a central unipolar and outer bipolar sections within the bundle.

A description of the model is given in Sec. II. In Sec. III we show that the distribution of particles along the lattice can exhibit a variety of phases depending on the system parameters. We show that, even for a low overall density of particles, particles can accumulate at minus ends as well as at plus ends. Moreover, we find a type of phase where density profiles of one type of particles can smoothly connect between low and high density. The density profiles for these different phases are well approximated by our mean-field analysis. In Sec. IV we consider how the MT switching rates $q_{1}$ and $q_{2}$ and the overall density $\Theta$ affect the contribution of each type of motor to the transport of cargos. Finally, in Sec. V we discuss the possible biological relevance and limitations of this model.

\section{LATTICE MODEL WITH ANTIPOLAR BUNDLING}

In this section we introduce our simple model of antipolar bundling of two MTs in the idealized cell [Fig. 1(a)]. The bundle (where we refer to each MT as a track) is modeled in Fig. 1(b) as a lattice of length $N$ with tracks of length $N-N_{1}$ and $N_{2}$, respectively, that overlap in a common section of length $N_{2}-N_{1}$. Each track has lanes that permit motion in each direction. The two plus ends of MTs are located at two ends of the lattice and the other ends of two MTs (the minus ends) are where the unipolar and bipolar sections meet. The two tracks are antipolar bundled so that there is a plus end at each extremity of the bundle. The middle section in the bundle is referred to as the bipolar section and the outer sections are referred to as the unipolar sections of relative length $x_{1}$ and $1-x_{2}$, respectively, where $x_{1,2}:=N_{1,2} / N$. Considering the symmetric organization of the MT bundle shown in [21], we assume henceforth a symmetric lattice with $x_{1}+x_{2}=1$.

The lattice supports motion of two types of cargo particle: Plus-type particles represent EEs that are driven by kinesin towards the plus end of the track, while minus-type particles represent EEs that are driven by dynein towards the minus end of the track. As the particles may have more than one motor
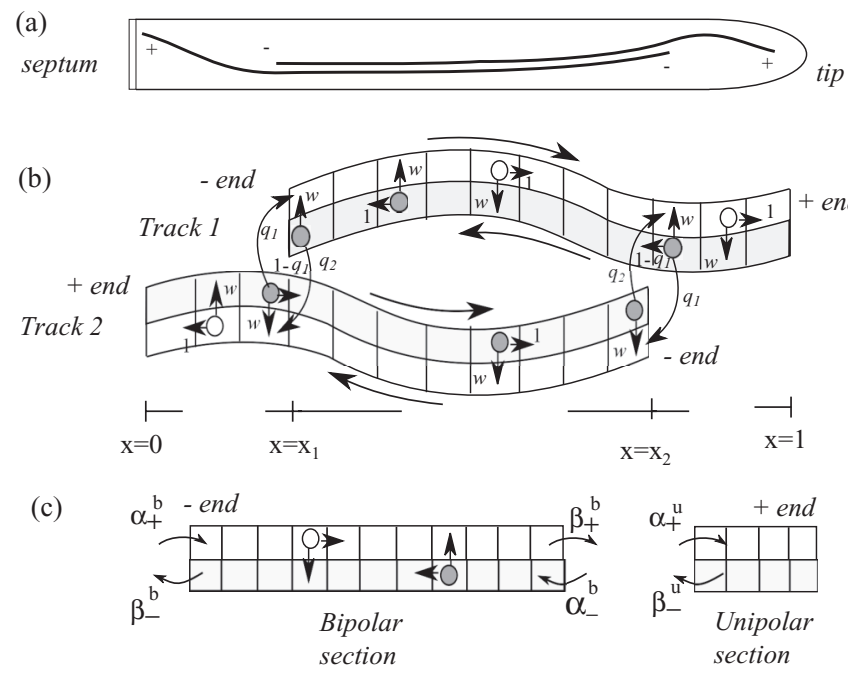

FIG. 1. (a) Schematic diagram of the hyphal tip cell of Ustilago maydis where two MTs with plus ends at cell poles (tip and septum) form an antipolar bundle. (b) Schematic diagram of bidirectional transport on the bundle modeled as a discrete lattice. The two tracks in the lattice represent two MTs of lengths $1-x_{1}$ and $x_{2}$ with (noflux) plus ends at two ends. Plus- (open) and minus-type (shaded) particles on the tracks move on separate lanes [open (shaded)] for particles moving forward to plus (minus) ends, respectively. Particles can change type (and thus lane) within the same track with rate $\omega$. The forward rate on each track is set to be 1, except at the junctions between the bipolar and unipolar sections. We assume that track switching occurs only at the junctions between sections and with rates $q_{1,2}\left(q_{1,2}=0\right.$ means that the MTs are uncoupled). (c) Assuming $x_{1}=$ $1-x_{2}$, we analyze steady solutions on the lattice (b) in terms of the densities on the bipolar and unipolar sections as shown, with effective boundary conditions that emerge via self-organization of the system.

type attached, they can change type and therefore direction when both motors bind. We assume that any case of opposing motors binding to the same track is resolved after a brief tug-of-war event [30-33] between the counteracting motors on the same particle. As collisions between opposite directed motion of EEs in U. maydis are rarely observed [21], which is similar to the dynein transport, we assume transport on each track in a particular direction is on a separate lane as in the two-lane model $[14,20]$. In summary, the lattice contains four lanes, each of which supports one direction and a single type of particle as illustrated in Fig. 1(b). We assume that particles remain attached [18,21] and the system is closed, i.e., there is no injection or exit of particles into or out of the lattice at the extremities of the bundle. The resulting particle number conservation during the transport means, for example, that once the end of a track is reached, a particle will remain there until it changes direction and continues motility back into the bundle.

We make further simplifying assumptions on track switching of particles, based on experimental observations of EE motility. In [21] it was shown that EEs carried by dynein can switch to be kinesin carried during the transport without reversal of transport direction. This implies that the motor type can change without reversing direction as well as there being a track switching and type change of EEs. It also demonstrates the bipolar structure of MT bundles in $U$. maydis hyphae. Moreover, [21] found that EEs often paused (in association 
with MT switching) near MT minus ends and suggested that minus ends are obstacles for EEs. In our model, the antipolar bundle of tracks means it is possible for a particle to switch between tracks with a change of type, without reversing transport direction. We assume track switching only occurs on passing junctions associated with minus ends of either MT. This simplification means we exclude the possibility of track switching for plus-type particles. These minimal assumptions on track switching are sufficient to allow particles to travel across the entire lattice from one extremity to the other. Because of the assumed symmetry $x_{1}+x_{2}=1$ we can reduce the lattice to Fig. 1(c), where the motion occurs independently on the bipolar and unipolar sections and the junctions can be thought of as imposing effective boundary conditions through self-organization of the system at the junctions.

We identify a location in the lattice by $(i, \ell, \pm)$, where $i \in$ $\{0, \ldots, N-1\}$ denotes the site, $\ell \in\{1,2\}$ denotes the track, and \pm denotes the lane direction on the track. Particles hop from one location to another with a certain transition rate where the possible transitions we consider are listed below.

(i) Forward motion. Plus- and minus-type particles can move forward by one location along a given lane. We assume equal rates for forward motion of plus- and minus-type particles (justified because of the similar velocities observed for in vivo transport [18,19]) and we assume homogeneous rates on each lane except at the junctions $\left(N_{2}, 1,-\right)$ and $\left(N_{1}-1,2,-\right)$ (for minus-type particles). For convenience, we set all forward rates to be $p=1$ (equivalently, we give all other rates in units of $p$ ).

(ii) Track switching. We allow minus-type particles at junctions $\left(N_{2}, 1,-\right)$ and $\left(N_{1}, 1,-\right)$ to step forward onto the second track (together with a type change) with rates $q_{1} \in[0,1]$ (obstacle-induced switching rate) and $q_{2} \in[0,1]$ (end-induced switching rate), respectively. Similarly, minus-type particles at $\left(N_{1}-1,2,-\right)$ and $\left(N_{2}-1,2,-\right)$ can switch onto the first track with the same obstacle-induced or end-induced rate. We assume that the total rate of forward motion at sites $\left(N_{2}, 1,-\right)$ and $\left(N_{1}-1,2,1\right)$ is still $p=1$.

(iii) Direction change. Plus- and minus- type particles can change direction on the same track and site by changing type (and therefore lane). We assume homogeneous and equal direction-change rates $w$ for all particles. We define $\Omega:=w N$, which characterizes the overall direction-change rate.

All transitions are assumed to be independent and instantaneous and are subject to a simple exclusion principle. This means that there can be at most one particle at each location $(i, \ell, \pm)$ of the lattice, and the presence of a particle at one location prevents other particles from moving into that location.

To describe the time evolution of this stochastic bidirectional transport process we write the system state using

$$
\tau_{i, \pm}^{\ell}(t) \in\{0,1\}
$$

where $1(0)$ denotes occupancy (vacancy) at location $(i, \ell, \pm)$ and time $t$; the state changes according to the above transition rates and the exclusion principle. The exclusion principle ensures that $\tau_{i, \pm}^{\ell}(t) \in\{0,1\}$ at all times. A special case is $q_{1}=q_{2}=0$, where the transport on each of the two tracks is independent of the other and the process is no longer ergodic. There is an extra symmetry in this case that involves interchanging the plus- and minus- type particles. This is caused by the assumption of equal forward-motion and turning rates, but in general there is no symmetry between the particle types. For the ergodic cases, the statistically stationary state will be independent of initial condition. The symmetric lattice structure gives a symmetric distribution of particles on two tracks where the mean number of particles on each track is equal.

For a statistically stationary state, the density (mean occupancy) of plus- and minus-type particles at location $(i, \ell, \pm)$ is

$$
\rho_{i}^{l}:=\left\langle\tau_{i,+}^{l}\right\rangle, \quad \sigma_{i}^{l}:=\left\langle\tau_{i,-}^{l}\right\rangle
$$

where $\langle\cdot\rangle$ denotes the ensemble (or time) average. These densities are related to the overall density $\Theta$ of particles by

$$
\Theta=\Theta_{+}+\Theta_{-}=\frac{\sum_{i=0}^{N-1}\left(\rho_{i}^{1}+\rho_{i}^{2}\right)}{2\left(N-N_{1}+N_{2}\right)}+\frac{\sum_{i=0}^{N-1}\left(\sigma_{i}^{1}+\sigma_{i}^{2}\right)}{2\left(N-N_{1}+N_{2}\right)},
$$

where $\Theta_{ \pm}$are the overall densities of plus- and minus-type particles, respectively. As the system is closed, the overall density $\Theta$ is conserved under the time evolution even though $\Theta_{ \pm}$may not be conserved. Meanwhile, the mean current (the mean rate of stepping forward per unit time) at location $(i, \ell, \pm)$ is given by

$$
J_{i, \pm}^{\ell}=\left\langle\tau_{i, \pm}^{\ell}\left(1-\tau_{i \pm 1, \pm}^{\ell}\right)\right\rangle
$$

away from the junctions (in units of $p$ ). At the junctions between sections, the mean currents for minus-type particles are

$$
\begin{aligned}
J_{N_{2},-}^{1}= & \left(1-q_{1}\right)\left\langle\tau_{N_{2},-}^{1}\left(1-\tau_{N_{2}-1,-}^{1}\right)\right\rangle \\
& +q_{1}\left\langle\tau_{N_{2},-1}^{1}\left(1-\tau_{N_{2}-1,+}^{2}\right)\right\rangle
\end{aligned}
$$

and

$$
J_{N_{1},-}^{1}=q_{2}\left\langle\tau_{N_{1},-}^{1}\left(1-\tau_{N_{1}-1,+}^{2}\right)\right\rangle,
$$

with similar expressions for junctions at the second track. On average, the net current in the unipolar section is zero and at the junction between sections it is balanced: $J_{N_{2},-}^{1}=J_{N_{2}-1,+}^{1}+$ $J_{N_{2}-1,-}^{2}$; however, there may be a nonzero net current in the bipolar section.

We believe that exact analytical solutions of the density profiles in our model with general parameters cannot be found using methods such as matrix products [6] or a Bethe ansatz [34] and therefore we use a combination of mean-field approximations and numerical simulations to understand the statistical behavior of the model.

For numerical simulation of the continuous-time discretestate model, we use the Gillespie algorithm [35]. Parameters that govern bidirectional transport in $U$. maydis are used where known from experiments; for example, we use $x_{1}=1-x_{2}=$ 0.2 , as microtubule minus ends in $U$. maydis hyphae are shown to be approximately uniformly distributed in the middle at about 10 in hyphal length away from cell poles where the MT minus ends are almost absent [21]. For the parameter $\Omega$, in vivo experiments in $[18,19]$ suggest a range of run length of order $10-70 \mu \mathrm{m}$ in a hyphal length of $L=100 \mu \mathrm{m}$, which gives [36] $\Omega \in[1,10]$. A lattice length of $N=500$ is used for simulations unless otherwise stated. We choose an initial condition where each track possesses an equal number of randomly distributed 
particles and run the simulation for at least $80000 \mathrm{~s}$, checking that the system reaches a statistically steady state by examining the convergence of finite-time averages.

\section{A. Particle-hole symmetry of the system}

The standard unidirectional ASEP on a single lane and the two-lane ASEP developed by Juhász [14] share a common feature: a particle-hole symmetry. Although this holds away from the junctions, at the junction there is no such exact symmetry. However, by adjusting the forward stepping rate of plus-type particles when crossing the junctions between tracks [i.e., an inhomogeneity in forward-motion rates for plus-type particles at locations $\left(N_{2}-1,1,+\right)$ and $\left.\left(N_{1}, 2,+\right)\right]$, the model can be adapted to give an exact particle-hole symmetry as explained below.

Note that particles are of two types and we divide holes in the lattice into two types. Minus-type holes refer to the holes in lanes for plus-type particles (as the holes move to the minus end). Similarly, plus-type holes refer to the holes in lanes for minus-type particles. A track switching of a minus-type particle from the unipolar to bipolar section corresponds to a track switching of a plus-type hole in the other direction, while a track switching of a minus-type particle from the bipolar to unipolar section corresponds to a track switching of a plus-type hole in the other direction.

If we adjust the forward rate for plus-type particles when crossing the junction at locations $\left(N_{2}-1,1,+\right)$ and $\left(N_{1}, 2,+\right)$ to be $\hat{p}=1-q_{2}$, then the system possesses the particle-hole symmetry, i.e., the system is invariant under the exchange

$$
\left(q_{1}, q_{2}, \tau_{i, \pm}^{\ell}\right) \leftrightarrow\left(q_{2}, q_{1}, 1-\tau_{i, \pm}^{\ell}\right) .
$$

This clearly maps $\left(\rho_{i}, \sigma_{i}\right)$ to $\left(1-\sigma_{i}, 1-\rho_{i}\right)$ and so $\Theta$ to $1-\Theta$. Note that the uncoupled system with homogeneous rates for plus-type particles (i.e., $q_{1,2}=0$ and so $\hat{p}=1$ ) is a special case with exact particle-hole symmetry. For $q_{1,2}$ small, the fact that $\hat{p} \approx 1$ means we still expect an approximate particle-hole symmetry. In later sections, we focus on low and intermediate overall densities and at least for $q_{1}$ and $q_{2}$ small, possible phases for high overall densities can be deduced from this approximate symmetry.

\section{STATIONARY DISTRIBUTION OF PARTICLES AND MEAN-FIELD ANALYSIS}

The system we consider is closed, but we can think of it as two half-closed unipolar end sections and two bipolar middle sections. For the spatially symmetric case $x_{1}=1-$ $x_{2}$ we assume the densities in the two unipolar (and bipolar, respectively) track sections are the same and so can effectively reduce this to a single bipolar and a single unipolar track as shown in Fig. 1(c). Through self-organization of the system, a statistically stationary solution will set up effective boundary rates (i.e., injection rates $\alpha_{ \pm}^{u(b)}$ and exit rates $\beta_{ \pm}^{u(b)}$ ) in Fig. 1(c) and we can use these to perform an analysis as in [14,20] to find the density profiles for statistically stationary states, given knowledge of the densities at the junctions.

For a lattice of length $N$, consider a rescaled position variable defined as $x=i / N \in[0,1]$ and in the continuum limit $N \rightarrow \infty$ we express $J_{ \pm}^{\ell}(x)=J_{i, \pm}^{\ell}$ as the (mean) unidirectional current and $J^{\ell}(x):=J_{+}^{\ell}(x)-J_{-}^{\ell}(x)$ as the net (mean) current. Moreover, we reexpress the densities on each track as $\rho^{\ell}(x)$ and $\sigma^{\ell}(x)$ for plus- and minus-type particles, respectively. The overall density of plus- and minus-type particles can then be reexpressed as

$\Theta_{+}=\frac{\int_{0}^{1}\left[\rho^{1}(x)+\rho^{2}(x)\right] d x}{2\left(1-x_{1}+x_{2}\right)}, \quad \Theta_{-}=\frac{\int_{0}^{1}\left[\sigma^{1}(x)+\sigma^{2}(x)\right] d x}{2\left(1-x_{1}+x_{2}\right)}$.

In the spatially symmetric lattice with $x_{1}+x_{2}=1$ for $x \in\left[x_{1}, 1\right]$ we write $\rho(x):=\rho^{1}(x)=\rho^{2}(1-x)$ and $\sigma(x):=$ $\sigma^{1}(x)=\sigma^{2}(1-x)$ so, for example, the overall density (including both types of particles) can be expressed as

$$
\Theta=\frac{\int_{x_{1}}^{1} \rho(x)+\sigma(x) d x}{2\left(1-x_{1}\right)} .
$$

The density profile in the statistically stationary state for plus- and minus-type particles in one section of the lattice is then governed by the following equations (ignoring the second derivative and second order of $1 / N$ ) and appropriate boundary conditions (see $[14,20]$ for details):

$$
\begin{aligned}
& 0=(2 \rho-1) \frac{d \rho}{d x}-\Omega(\rho-\sigma), \\
& 0=(1-2 \sigma) \frac{d \sigma}{d x}+\Omega(\rho-\sigma) .
\end{aligned}
$$

Meanwhile, the unidirectional currents in each direction on this section are

$$
J_{+}(x)=\rho(x)[1-\rho(x)], \quad J_{-}(x)=\sigma(x)[1-\sigma(x)]
$$

and the net current is

$$
J(x)=\rho(x)[1-\rho(x)]-\sigma(x)[1-\sigma(x)] .
$$

Taking the sum of (1), note that

$$
\frac{d J(x)}{d x}=\frac{d}{d x}[\rho(1-\rho)-\sigma(1-\sigma)]=0,
$$

which implies that there is a constant net current $J(x)=J_{0}$ for the mean-field approximation in the stationary state in each section. A positive (negative) net current indicates a net current towards the plus (minus) end.

\section{A. Spatial distribution in the unipolar section}

As the unipolar section of the first track $\left(x_{2}<x \leqslant 1\right)$ is closed at one end, this corresponds to boundary conditions $\rho(1)=1$ and $\sigma(1)=0$ and we assume that the end $x=x_{2}$ of the unipolar section is associated with injection and exit rates $\alpha_{+}^{u}$ and $\beta_{-}^{u}$ as in Fig. 1(c). In this section we analyze the density profiles in the unipolar section for general $\alpha_{+}^{u}$ and $\beta_{-}^{u}$. As discussed in [20], the unipolar section has zero net current due to the closed boundary at the right end. Thus $J=\rho(x)[1-$ $\rho(x)]-\sigma(x)[1-\sigma(x)]=0$, leading to two possible solutions

$$
\rho(x)=1-\sigma(x), \quad \rho(x)=\sigma(x) .
$$

Hence, solving the ordinary differential equation (ODE) (1), there are two possible solutions: complementary density

$$
\rho(x)=1-\sigma(x)=1-\Omega+\Omega x
$$

and equal density $\rho(x)=\sigma(x)=C$. The boundary conditions at $x=1$ mean that a complementary density region starts from 
the plus end and extends toward the interior of the unipolar section, while an equal density region can appear away from the end.

For low injection and high exit rates, the constant in the equal density case is $C=\rho\left(x_{2}\right)=\alpha_{+}^{u}$. The density profile of plus-type particles exhibits a shock between equal density and complementary density regimes, while the density of minus-type particles is continuous except near the left end. We refer to this as an SL phase; the first letter refers to plus-type particles, while the second one refers to minus-type particles: S stands for shock, while L stands for low (less than 1/2). By the approximate particle-hole symmetry, analogous to the SL phase, there is an HS phase where the equal density is over one-half and minus-type particles experience a shock.

For small enough turning rate $\Omega$, the shock location $x_{s}$ can be found by matching $\lim _{x \rightarrow x_{s}^{-}} \rho(x)=\lim _{x \rightarrow x_{s}^{+}} 1-\rho(x)$ between the two regions, meaning $x_{s}=1-\frac{\alpha_{+}^{u}}{\Omega}$ as long as $x_{2}<x_{s}<1$. If $1-\frac{\alpha_{+}^{u}}{\Omega} \leqslant x_{2}$ then the whole of the unipolar section is filled with the complementary density regime and we refer to this as an HL phase (H stands for high density).

In contrast, if the tuning rate $\Omega$ is high enough, a region of maximal current with a density of $1 / 2$ can appear near the open end of the unipolar section for high enough injection rate. This is referred as an MM phase (M stands for maximal unidirectional current) and only occurs when $1-\Omega+\Omega x_{2}<$ $1 / 2$, i.e., if $\Omega x_{1}>1 / 2$.

In summary, the possible phases of density profiles for the unipolar section with general left boundary conditions $\alpha_{+}^{u}$ and $\beta_{-}^{u}$ are shown in Fig. 2 for both $\Omega x_{1}<1 / 2$ and $\Omega x_{1}>1 / 2$. On the coexistence line $\alpha_{+}^{u}=\beta_{-}^{u}$, we have $\rho\left(x_{2}\right)=\alpha_{+}^{u}=1-$ $\sigma\left(x_{2}\right)$ and note that $\rho\left(1-\frac{\alpha_{+}^{u}}{\Omega}\right)=1-\alpha_{+}^{u}$ in the complementary density, thus a similar discussion as in [14] can be applied in the region $\left[x_{2}, 1-\frac{\alpha_{+}^{u}}{\Omega}\right]$. In the next section we consider a similar approach to the bipolar section and hence on the entire bundle.

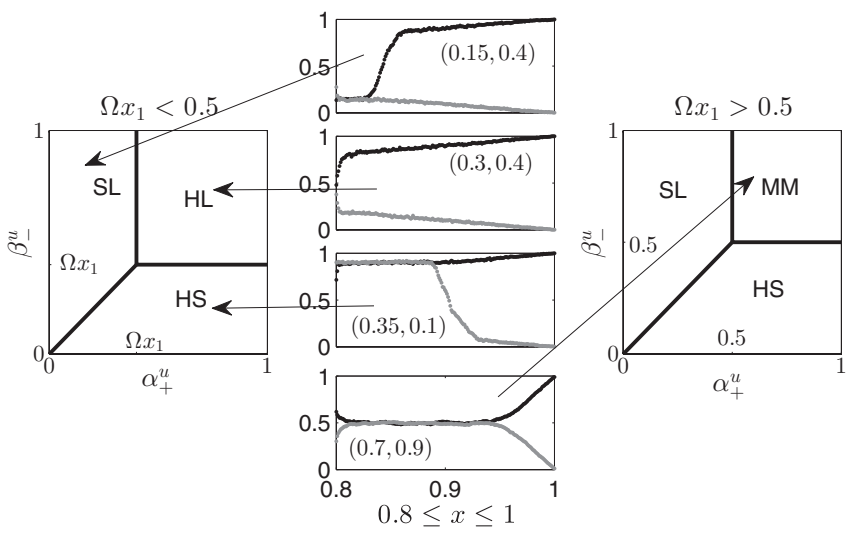

FIG. 2. Phase diagram of the density profile in the unipolar section with general left boundary conditions $\alpha_{+}^{u}$ and $\beta_{-}^{u}$ and closed right boundary conditions, for $\Omega x_{1}<1 / 2$ (left panel) and $\Omega x_{1}>1 / 2$ (right panel), where $x_{1}=1-x_{2}=0.2$. Numerical examples of density profiles in the unipolar section for each phase are illustrated with indicated boundary conditions $\left(\alpha_{+}^{u}, \beta_{+}^{u}\right)$. The top three density profiles in the middle column use $\Omega=1$, while the bottom density profile is using $\Omega=10$. In the density profiles, black dots are for plus-type particles, while gray dots are for the minus type.

\section{B. Analysis of spatial distribution along the entire bundle}

We now consider the density profiles in the entire symmetric bundle. In the uncoupled case $\left(q_{1}=q_{2}=0\right)$, the stochastic process on each track is exactly the same as the two-lane ASEP discussed in [14] assuming equal direction-change rates and no-flux boundary conditions. The density profile on the first track consists of three segments in general; for low overall density, an equal-density segment occurs in the middle and this is surrounded by complementary-density segments at the ends of the track.

For either $q_{1}$ or $q_{2}$ positive, we analyze the density profiles by considering two ASEPs on the first track with corresponding boundary rates in each section. The boundary rates are set by self-organization of the system and depend on densities in the other sections. The density profiles can exhibit a variety of phases even if the parameters $\Omega$ and $\Theta$ are fixed. In the following subsections we show that a mean-field approximation with appropriate boundary conditions agrees well with numerical simulations for a variety of density profiles and phases.

In the generic case where $q_{1}, q_{2}>0$, we can assume that the net current $J$ on a single track in the bipolar section is not necessary zero. For a small net current there are nearly-equaland nearly-complementary-density solutions $\sigma \approx \rho$ and $\sigma \approx$ $1-\rho$ (see Appendix A for details). Having neither $\rho$ nor $\sigma$ close to $1 / 2$, i.e., assuming

$$
\min \{1-\rho, \rho\}<\epsilon, \quad \min \{1-\sigma, \sigma\}<\epsilon
$$

for some small $\epsilon$, gives a net current $J=O(\epsilon)$ from Eq. (2). Furthermore, we approximate the constant net current $J=$ $\rho(1-\rho)-\sigma(1-\sigma)=(\rho-\sigma)(1-\rho-\sigma)$ by

$$
\begin{aligned}
& J=\operatorname{sgn}(1-2 \rho)(\rho-\sigma)+O\left(\epsilon^{2}\right), \\
& J=\operatorname{sgn}(1-2 \rho)(\rho+\sigma-1)+O\left(\epsilon^{2}\right)
\end{aligned}
$$

for nearly-equal- and nearly-complementary-density regions, respectively. The solutions of density profiles for plus-type particle [see (A1)] can thus be approximated [with error $O\left(\epsilon^{2}\right)$ ] as

$$
\rho=-J \Omega x+C_{e}, \quad \rho=\Omega x+C_{c}
$$

for nearly equal and nearly complementary densities, respectively, and the corresponding densities of minus-type particles can be approximated as

$$
\sigma=\rho+\operatorname{sgn}(2 \rho-1) J, \quad \sigma=1-\rho-\operatorname{sgn}(2 \rho-1) J .
$$

The constants $C_{e(c)}$ in (5) are functions of the boundary rates $\alpha_{ \pm}^{b}$ and $\beta_{ \pm}^{b}$ that are set by self-organization in the closed system, though for low $J$ we are able to successfully estimate the dependence on some of the parameters. Note that a minus-type particle crossing the junction $x=x_{2}$ from the unipolar to the bipolar section switches to the other track with rate $q_{1}$ (which contributes to the injection of plus-type particles on the other track in the bipolar section) and keeps on the same track with rate $1-q_{1}$ (which contributes to the injection of minus-type particles). Therefore, by the spatial symmetry [which gives $\sigma^{1}\left(x_{2}\right)=\sigma^{2}\left(x_{1}\right)$ ], we approximate the injection rates

$$
\alpha_{+}^{b}=\sigma\left(x_{2}\right) q_{1}, \quad \alpha_{-}^{b}=\sigma\left(x_{2}\right)\left(1-q_{1}\right) .
$$


Moreover, a minus-type particle on the second track switches to the first track with rate $q_{2}$ when crossing the junction at $x=x_{2}$ (which contributes to the exit of minus-type particles in the bipolar section), a plus-type particle steps forward with rate 1 when crossing the junction (which contributes to the exit of plus-type particles), and both the minus- and plus-type particles share the same target site when they move. Thus, by spatial symmetry, we have $\beta_{+}^{b}+\beta_{-}^{b}=1-\rho\left(x_{2}\right)$ and $\beta_{-}^{b}=$ $q_{2} \beta_{+}^{b}$. This gives exit rates

$$
\beta_{+}^{b}=\frac{1-\rho\left(x_{2}\right)}{1+q_{2}}, \quad \beta_{-}^{b}=\frac{\left[1-\rho\left(x_{2}\right)\right] q_{2}}{1+q_{2}} .
$$

The boundary rates in (7) and (8) determine the constant in nearly-equal- and nearly-complementary-density approximation [see (A1)]. The following sections use the density approximation in (A1) and (5), assuming low net current and neither $\rho$ nor $\sigma$ close to $1 / 2$, to obtain approximate density profiles along the entire lattice.

\section{Phases for low overall densities}

For low overall density $\Theta \ll 1 / 2$, the unipolar sections must have low density and thus the unipolar section will be in an SL phase, where densities $\rho(x)=\sigma(x)=\bar{\sigma}$ are equal and constant away from the plus end. There will be a shock at location $x_{s}^{u}=1-\bar{\sigma} / \Omega$ in the unipolar section from (3). When both types of particles in the bipolar section have low density, these densities are determined by the injection rates $\alpha_{ \pm}^{b}$ in (7) with $\sigma\left(x_{2}\right)=\bar{\sigma}$. Thus, in the bipolar section, the density profiles are governed by the parameters $\Omega, q_{1,2}$, and $\bar{\sigma}$. If $q_{2}=0$ then queueing appears at the minus ends in a similar manner to the queueing at the plus end in the unipolar section. By contrast, for a sufficiently large $q_{2}$ any queuing particles at the minus end are expected to move into the unipolar section of another track. This leads to low densities for both types of particles in the bipolar section and we refer to it as an LL-SL phase for the entire system; the two letters (LL in this case) before the hyphen refer to the bipolar section, while the other two (SL in this case) refer to the unipolar section.

Explicit expressions of the mean-field approximation for the density profiles can be found using the nearly-equaldensity approximations (5) and (6) for the bipolar section and conservation of $\Theta$. A detailed calculation of these expressions are given in Appendix B, while Fig. 3(a) shows that the meanfield approximations agrees well with numerical simulations for this LL-SL phase. This LL-SL phase is lost for lower $q_{1,2}$ as an additional boundary layer may arise near the junctions; a shock of minus-type particles can form near the minus end. We refer to this case as an LS-SL phase (i.e., in the bipolar section minus-type particles are in shock state and plus-type particles are in low density, while in the unipolar section a shock forms for the plus type and the density of the minus type is low) [see Fig. 3(b)]. In the mean-field approximation, a shock for minus-type particles in the bipolar section stabilizes at $x=x_{s}^{b}$ where

$$
\lim _{x \rightarrow x_{s}^{b^{+}}} \sigma(x)=1-\lim _{x \rightarrow x_{s}^{b^{-}}} \sigma(x) .
$$

The density $\rho\left(x_{2}\right)=\bar{\sigma}$ can also be used to give the approximate boundary rate $\beta_{-}^{b}$ in (8) in terms of the parameters, using
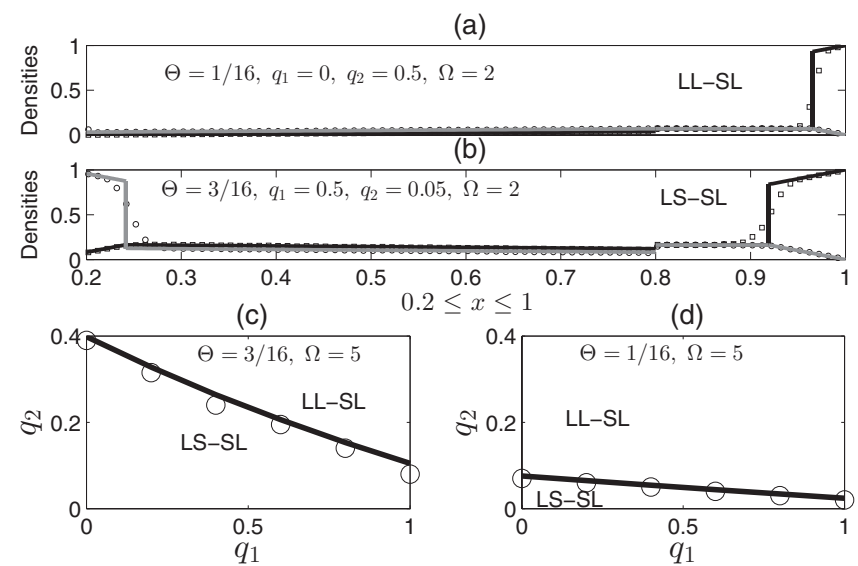

FIG. 3. Panels (a) and (b) show density profiles for LL-SL and LS-SL phases on the first track with $x_{1}=1-x_{2}=0.2$ and other parameters indicated. For plus-type (minus-type) particles, black (gray) lines show the density solution from mean-field approximations and squares (circles) show the averaged densities (over time interval $T=60000 \mathrm{~s}$ ) using a Gillespie algorithm. Panels (c) and (d) show the transition between LL-SL and LS-SL phases for indicated $\Omega$ and $\Theta$. Lines are from the mean-field approximation (10), while circles are from numerical simulations; a shock is identified if $\rho_{N_{1}+1}^{1}>0.5$.

approximations of nearly equal and nearly complementary densities (5) and (6) for right and left sides of the shock in the bipolar section. Figure 3(b) shows that the mean-field approximation agrees well with numerical simulations for this phase.

The transition between LS-SL and LL-SL phases for low overall density involves the shock in the bipolar section meeting the junction between sections i.e., $x_{s}^{b} \rightarrow x_{1}$. In other words, the nearly equal density of minus-type particles $\sigma(x)$ in (B2) satisfies $\lim _{x \rightarrow x_{1}} \sigma(x)=1-\sigma\left(x_{1}\right)=\beta_{-}^{b}$, which gives

$$
\frac{q_{2}(1-\bar{\sigma})}{1+q_{2}}=\bar{\sigma} q_{1}-\frac{\bar{\sigma}\left(2 q_{1}-1\right)}{\Omega\left(x_{2}-x_{1}\right)+1} .
$$

Recall that $\bar{\sigma}$ is related to the overall density $\Theta$ by (B5). Figures 3(c) and 3(d) show examples of this prediction on the borderline between LL-SL and LS-SL phases in the space $\left(q_{1}, q_{2}\right)$ against numerical simulations where a shock is identified by over one-half density of minus-type particles at the penultimate site to the minus end. Qualitatively, this prediction agrees well with the simulations.

\section{Phases for intermediate overall densities}

For intermediate overall densities, the system displays a wide variety of phases for different parameter values. We have not attempted to characterize all the possible phases for this intermediate density, but we do investigate some of these phases in detail: one with shocks of both types of particles and a second with a smooth connection in the bipolar section.

\section{The SS-HL phase}

Note that a small $\Omega$ could cause the unipolar section to be in an HL phase with shocks for both types of particles in the bipolar section. This is referred as an SS-HL phase; 


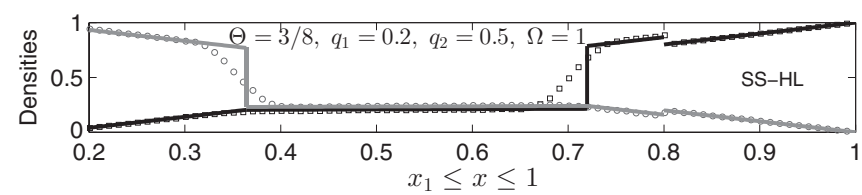

FIG. 4. Density profile on the first track with parameters indicated shows an SS-HL phase. The markers for simulation and mean-field approximations are as in Figs. 3(a) and 3(b).

see Fig. 4 for an example. In this phase, the density profiles have nearly equal density in the middle separating two nearlycomplementary-density regimes in the bipolar section; the four boundary conditions $\alpha_{ \pm}^{b}, \beta_{ \pm}^{b}$ given in (7) and (8) are all satisfied. Therefore, we have

$$
\begin{aligned}
& \rho\left(x_{1}\right)=\alpha_{+}^{b}<1 / 2, \quad \rho\left(x_{2}\right)=1-\beta_{+}^{b}, \\
& \sigma\left(x_{1}\right)=1-\beta_{-}^{b}, \quad \sigma\left(x_{2}\right)=\alpha_{-}^{b}<1 / 2 .
\end{aligned}
$$

If $\rho$ and $\sigma$ in the bipolar section are not close to $1 / 2$, then from (4) we approximate the net current by $J=\beta_{+}^{b}-$ $\alpha_{-}^{b}=\alpha_{+}^{b}-\beta_{-}^{b}$. We also approximate $\rho\left(x_{2}\right)$ and $\sigma\left(x_{2}\right)$ in the boundary rates by the limit in the complementary density (3) as $x \rightarrow x_{2}$, i.e., $1-\rho\left(x_{2}\right) \approx \sigma\left(x_{2}\right) \approx x_{1} \Omega$. Thus the net current is approximated by

$J=\beta_{+}^{b}-\alpha_{-}^{b}=\alpha_{+}^{b}-\beta_{-}^{b}=\left(\frac{1}{1+q_{2}}-1+q_{1}\right) x_{1} \Omega$.

Similar to the previous discussion, for the SS-HL phase the relation between the shock locations in the bipolar section and the overall density can in principle be found using (5) and (6) to give approximate density profiles in the bundle. Figure 4 shows that the approximated solution from the mean field agrees well with numerical simulations.

Note that the approximation (11) of the net current suggests that the direction of the net current is governed by $q_{1,2}$ and moreover that $\left(1-q_{1}\right)\left(1+q_{2}\right)=0$ gives a zero net current. We expect constant density in a region of the bipolar section when there is zero net current, as in Sec. III A. Particularly when the overall density $\Theta=1 / 2$, we find a maximum unidirectional current region in the bulk where $\rho^{1}(x)=\sigma^{1}(x)=$ $1 / 2$. This region can be estimated by identifying degenerated shocks (with zero shock height) for both types of particles, which can be approximated as $\left[\frac{1}{2 \Omega}+\left(1-q_{1}\right) x_{1}, 1-\frac{1}{2 \Omega}-\right.$ $\left.q_{1} x_{1}\right]$. Note also that this maximum unidirectional current can appear with an MM phase in the unipolar section.

\section{Phases with smooth connection}

For a nonzero net current $J$, the nearly equal density solution is a monotonic function in position seen from (A1). Thus, simply by increasing the overall density from a low value, the maximum value of the density profiles in the nearly-equal-density region would increase and eventually reach $1 / 2$. Figure 5 shows density profiles together with a plot of $\rho$ vs $\sigma$ under different $\Theta$; particularly for $\Theta=5 / 16$ and $9 / 16$, the nearly-equal-density regions contain both high and low densities and one of the types (the plus type in this example) exhibits slower change between low and high density than the other type. Moreover, Fig. 6 compares the density profiles in different system sizes where the density profile
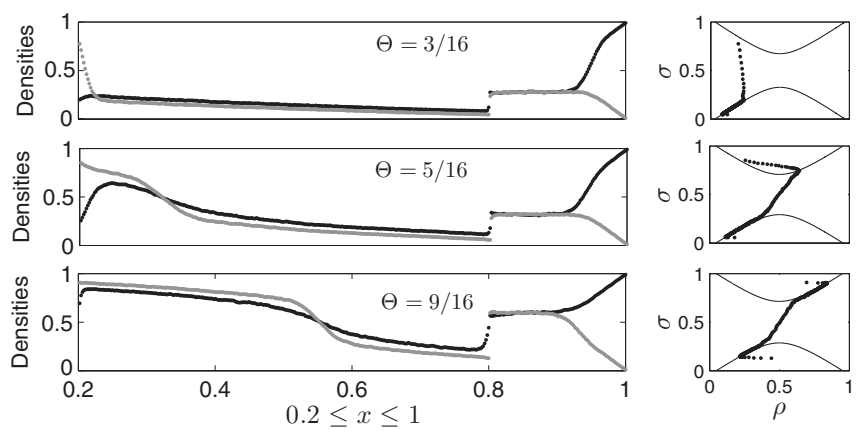

FIG. 5. On the left are density profiles on the first track for four different overall densities as indicated; dark lines are for plus-type particles, while gray lines are for minus-type particles. On the right is a plot of $\rho$ vs $\sigma$ from the corresponding left panel; the solid lines show the relation $J=\rho(1-\rho)-\sigma(1-\sigma)$, where $J$ is the constant net current. Deviations of the dots from the solid lines are probably due to finite-size or boundary-layer effects. Other parameters are $\Omega=5, q_{1}=0.8$, and $q_{2}=0.2$.

in the middle is more shocklike for plus-type particles and remains almost unchanged for the other type.

This suggests that, for this example, in the bipolar section $\rho$ smoothly increases through $1 / 2$ with $x$ while $\sigma$ has a shock separating low and high densities. We say this phase has a smooth connection between low and high density for one type of particles and we denote it as an SC (or a CS) phase, depending on which type of particles exhibits a smooth connection (we ignore the existence of additional shocks for the type with a smooth connection in the bipolar section). The letter S stands for shock, while the letter C stands for connection; which type density smoothly connects is related to the sign of the net current $J$ : A positive $J$ is associated with plus-type particles having a smooth connection and vice versa.

If there is a CS (or an SC) phase in the bipolar section, there are various possible phases in the unipolar section; see Figs. 5 and 7, where the unipolar section can be seen to be in HS, HL, SL, or SS phases depending on parameters. Note that the SS phase in the unipolar occurs when the boundary rate $\alpha_{+}^{u}=\beta_{-}^{u}$. In contrast to the polynomial function of density profiles shown in [14] with symmetric open boundary condition, both density profiles exhibit shocks here due to the overall density being fixed; this is consistent with the discussion in [14]. Furthermore, the smooth connection phases are generic in the parameter space $q_{1,2}$ [seen from Fig. 8 where each frame

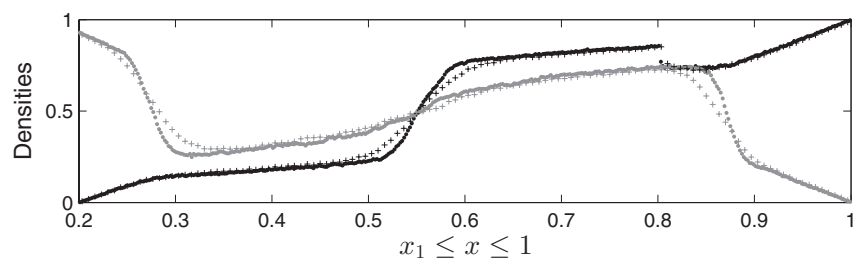

FIG. 6. Density profiles on the first track for system sizes $N=$ $300(+)$ and $N=1000(\bullet)$. Black shows $\rho$, while gray shows $\sigma$. The shock width near $x=0.55$ for $\rho$ is shorter for the larger system size, while $\sigma$ shows a smooth connection through $\sigma=1 / 2$. Other parameters are $\Omega:=\omega N=5, q_{1}=0, q_{2}=0.5$, and $\Theta=1 / 2$. 
(a)

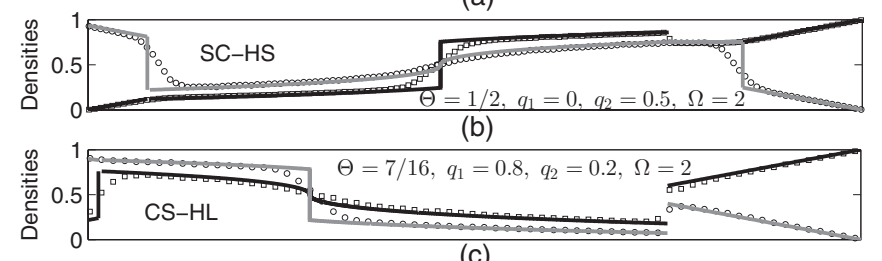

(c)

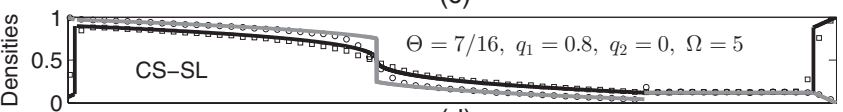

(d)

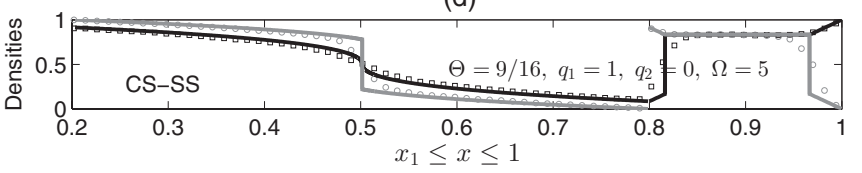

FIG. 7. Density profiles on the first track with indicated parameters showing smooth connection phases for intermediate overall densities. A CS phase in the bipolar section indicates that minus-type particles exhibit a smooth connection through density $1 / 2$ at a point where plus-type particles exhibit a shock, while for an SC phase the plus-type particles exhibit the smooth connection. The markers for simulation and mean-field approximations are as in Figs. 3(a) and 3(b).

represents the density profiles for $\rho(x)$ (left panel) and $\sigma(x)$ (right panel) via color for fixed $q_{1}$ and changing $q_{2}$ by every 0.1 between 0 and 1$]$. The parameter $q_{1} \in\{0,0.2,0.5,0.8,1\}$ is increased from the top frame to the bottom frame in both panels. In addition, there are both SC and CS phases in the parameter space $\left(q_{1}, q_{2}\right)$. For instance, plus-type particles exhibit a smooth connection for high $q_{1}$, while minus-type particles exhibit this for low $q_{1}$.

The density profiles in smooth connection phases can also be understood by mean-field approximations. When using approximation (A1) for densities in the bipolar section, the constants can in principle be worked out by considering the fact of the overall density conservation, similar to the previous discussion on the LL-SL phase. However, due to the nonlinearity in the expression (A1), we examine the mean-field approximation for the CS (or SC) phase by choosing appropriate $J$ and boundary conditions to satisfy the
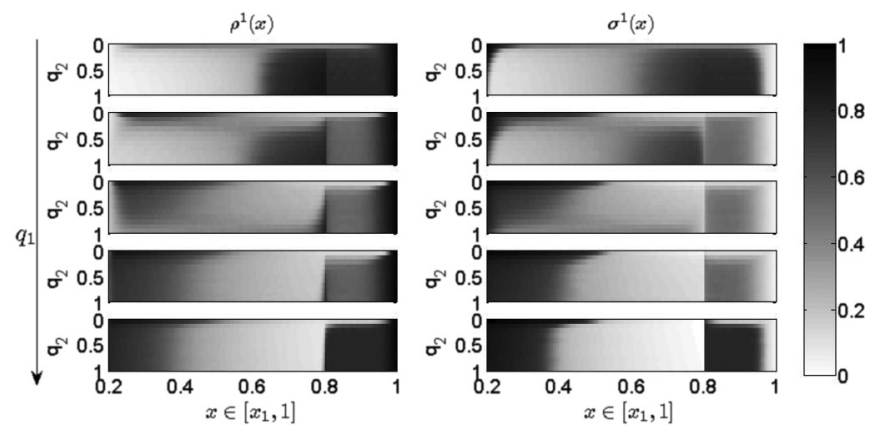

FIG. 8. A variety of phases arise in the bundle for fixed $\Theta=7 / 16$ and $\Omega=5$ when varying the switching rates $q_{1,2}$. Each frame in the left and right panels represents densities of plus- and minus-type particles for fixed $q_{1}$ when varying $q_{2}$ by every 0.1 between 0 and 1 . The fixed parameter $q_{1} \in\{0,0.2,0.5,0.8,1\}$ increases from the top to bottom frame in both panels. overall density $\Theta$ and the densities in the unipolar section. Take Fig. 7(a) as an example where minus-type particles smoothly connect low and high densities and also form a shock in the bipolar section, and in the unipolar section densities are in an HS phase with an equal high density that is constant away from the plus end, say, $\bar{\sigma}$. In the CS-HS phase, given a net current $J$ and $\bar{\sigma}$ with boundary conditions $\alpha_{+}^{b}=\bar{\sigma} q_{1}$ and $\alpha_{-}^{b}=\bar{\sigma}\left(1-q_{1}\right)$, we would have the nearly equal and nearly complementary densities by approximation (A1) in the bipolar section and would also have the equal and complementary densities from Sec. III A in the unipolar section. The parameters $J$ and $\bar{\sigma}$ are chosen in order to match the shock condition for the minus-type particle in the bipolar section as in (9) and the overall density $\Theta$. Particularly, in this example, the mean-field solution with $\bar{\sigma}=0.753$ and $J=-0.065$ satisfies the shock condition and the overall density; Fig. 7(a) shows the agreement between the mean-field approximation with chosen parameters and the simulation. Similar comparisons for smooth connection phases with an HL, SL, or SS phase in the unipolar section are shown in Figs. 7(b)-7(d).

\section{E. Phases for high overall densities}

When the system has high overall density, the vacancies are in low overall density. From the discussion of particlehole approximate symmetry in Sec. II, we can see that an HH-HS and SH-HS phase can appear. Moreover, note that the approximate symmetry may break when $q_{1}$ is large, in which case we find another phase where plus-type particles are in high density in both sections while minus-type particles exhibit a shock. We refer to this as an HS-HS phase (see Fig. 9). In this phase the boundary rates $\alpha_{-}^{b}$ and $\beta_{ \pm}^{b}$ aresatisfied and Fig. 9 shows that the mean-field approximation agrees with numerical simulations on the density profiles.

\section{DOMINANCE OF PARTICLES IN TRANSPORT}

Both types of particles are essential for bidirectional transport along the bundle of tracks in the unipolar section. However, the possibility of nonzero net flux already indicates that one type of particle is more responsible for the transport in the bipolar section. Indeed, in vivo experiments of EEs suggest that kinesin-3 is the main motor for long-range EE motility across an antipolar MT bundle [21]. In the following we discuss the contribution of each type of particle to the transport in terms of occupancy as well as current in our model.

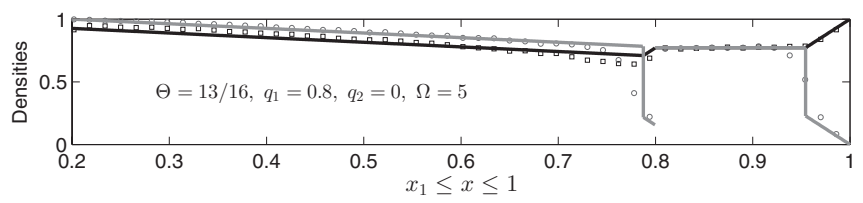

FIG. 9. Density profiles on the first track with indicated parameters show an HS-HS phase. The markers for simulation and mean-field approximations are as in Figs. 3(a) and 3(b). 


\section{A. Dominance in occupancy}

For the transport in the entire bundle, one way to quantify the dominance in occupancy is to estimate the fraction of plus-type particles in the entire bundle, $0 \leqslant F_{+} \leqslant 1$, where

$$
F_{+}:=\frac{\Theta_{+}}{\Theta}=\frac{\int_{x_{1}}^{1} \rho^{1}(x) d x}{2 \Theta\left(1-x_{1}\right)}
$$

for a symmetric lattice $x_{1}+x_{2}=1$. Recall that $\Theta$ is an overall density of particles in the bundle, which is preserved during the transport and so is considered as a parameter. Note that $F_{+}=1 / 2$ in the case $q_{1}=q_{2}=0$ as in this case the tracks decouple and the assumption of equal turning rates means that the densities have a symmetry $\rho(x)=\sigma(1-x)$.

Track switching is accompanied by a change of type from minus to plus, thus increasing either $q_{1}$ or $q_{2}$ is the potential to increase the number of plus-type particles and thus increase $F_{+}$. If $F_{+}$is close to 1 then plus-type particles are in significant dominance in terms of occupancy in the entire bundle. For
$\Omega=0$ and $q_{1,2}>0$ together with a sufficiently low overall density $\Theta$ we get close to the maximum $F_{+}=1$.

When considering the fraction of plus-type particles within each section, it is clear that in the unipolar section there are more plus-type than minus-type particles for any parameters. In contrast, the fraction in the bipolar section

$$
F_{+}^{b}:=\frac{\int_{x_{1}}^{x_{2}} \rho(x) d x}{\int_{x_{1}}^{x_{2}} \rho(x)+\sigma(x) d x}
$$

is not as easy to estimate for general overall density $\Theta$.

For low overall density, the system is in either the LL-SL or LS-SL phase and the mean-field approximation predicts the corresponding density profiles well. For sufficiently large $q_{2}$ [i.e., larger than the critical value satisfying (10)] there will be low densities for both types of particles in the bipolar section, approximately independent of $q_{2}$. Both fractions $F_{+}$and $F_{+}^{b}$ can be expressed using the approximated density profiles, ignoring boundary layers. Thus, in this phase the fraction in the entire bundle is approximately

$$
F_{+} \approx \frac{\frac{1}{2} \bar{\sigma} \Omega\left(x_{2}-x_{1}\right)^{2}+\bar{\sigma} q_{1}\left(x_{2}-x_{1}\right)+1-\frac{1}{2} \bar{\sigma}^{2} / \Omega+\left(x_{1}-\bar{\sigma} / \Omega\right) \bar{\sigma}}{\bar{\sigma} / \Omega+(1-2 \bar{\sigma} / \Omega) \bar{\sigma}}
$$

where $\bar{\sigma}$ is associated with $\Theta$ as in (B5), and the fraction in the bipolar section is

$$
F_{+}^{b} \approx \frac{1}{2}+\frac{2 q_{1}-1}{2 \Omega\left(x_{2}-x_{1}\right)+2} .
$$

Both fractions show an independence of $q_{2}$ and linear dependence on $q_{1}$. Comparatively, the latter expression for the fraction in the bipolar section is a simpler function of $\Omega$ and $q_{1}$ for fixed $x_{1,2}$. We can see that this expression agrees well with simulations from Fig. 10. Moreover, from this approximation, $q_{1}=1 / 2$ gives an approximately equal contribution in the bipolar section for each type of particles. This agrees with a

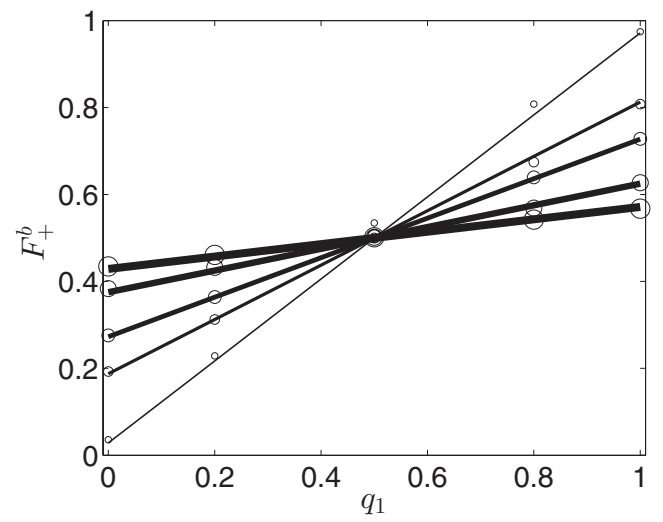

zero net current in (B3), which leads to equal density in an LL phase. Moreover, the sign of $q_{1}-1 / 2$ determines which type of particle is in dominance and by decreasing $\Omega$ the dominance can be enhanced. Particularly, for a small $\Omega$,

$$
\lim _{q_{1} \rightarrow 1} F_{+}^{b}\left(q_{1}\right) \approx 1, \quad \lim _{q_{1} \rightarrow 0} F_{+}^{b}\left(q_{1}\right) \approx 0
$$

That is to say, for sufficiently low $\Omega$, plus-type particles dominate in the bipolar section for sufficiently large switching rate $q_{1}$; in contrast, minus-type particles dominate for sufficiently small $q_{1}$.
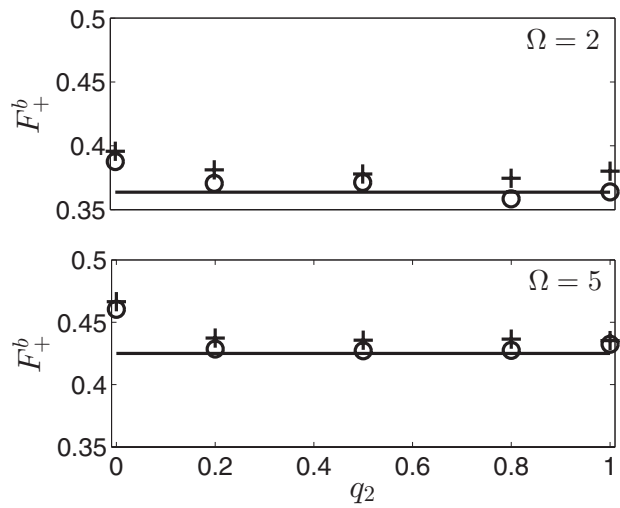

FIG. 10. Fraction of plus-type particles in the bipolar section. On the left the lines are from the approximated equation (13) for $\Omega \in$ $\{0.1,1,2,5,10\}$, with bolder lines for larger $\Omega$, while circles are for simulations, with larger circles for larger $\Omega$. Other parameters are $\Theta=1 / 16$ and $q_{2}=1$. The right panels examine the independence of this fraction on the overall density $\Theta$ and switching rate $q_{2}$ if sufficiently large; pluses and circles are for $\Theta=3 / 16$ and $1 / 16$, respectively, in both the top and bottom panels. Simulated data presented use $q_{1}=0.2$; quantitatively similar figures can be obtained for other $q_{1}$. 

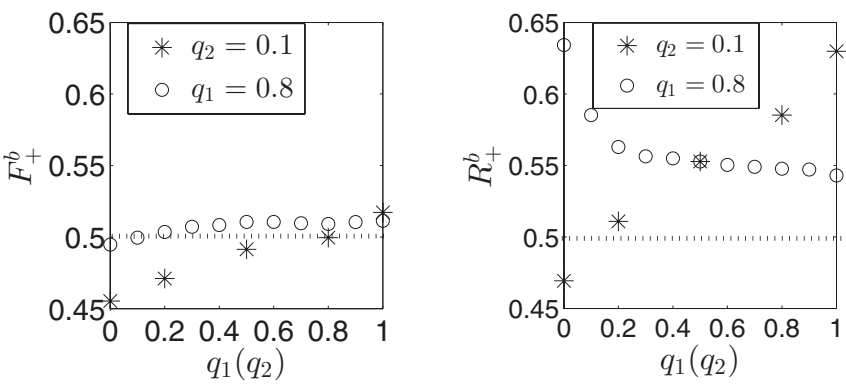

FIG. 11. Fraction of plus-type particles $F_{+}^{b}$ (left panel) and the ratio of the average current for plus-type particles $R_{+}^{b}$ (right panel) in the bipolar section with varying $q_{1}$ (marked by $*$ ) or $q_{2}$ (marked by + ) for $\Omega=5$ and $\Theta=7 / 16$. Simulated data presented use fixed $q_{1}=0.8\left(q_{2}=0.1\right)$ and varying $q_{2}\left(q_{1}\right)$; qualitatively similar figures can be obtained for other values of $q_{1,2}$.

For intermediate overall density, the plus-type and/or minus-type particles form shocks near the junctions between sections. In contrast to low overall densities where $F_{+}^{b}$ is highly dependent on $q_{1}$ and weakly dependent on $q_{2}$, in the intermediate overall densities, the fraction in the bipolar section $F_{+}^{b}$ shows a relatively weak dependence on both switching rates $q_{1,2}$. The left panel of Fig. 11 shows as an example with an overall density $\Theta=7 / 16$ of how the fraction in occupancy varies changes with the rates $q_{1,2}$.

\section{B. Dominance in current}

The directed currents $J_{ \pm}$are also important quantities to characterize the transport capacity: A large current indicates efficient transport. In contrast with the standard unidirectional ASEP on a single lane (where both the density and current are constant along the bulk of the lane), in our model neither the density nor the directed current of each type of particles on a single track is constant (see Fig. 12 for examples). Thus we
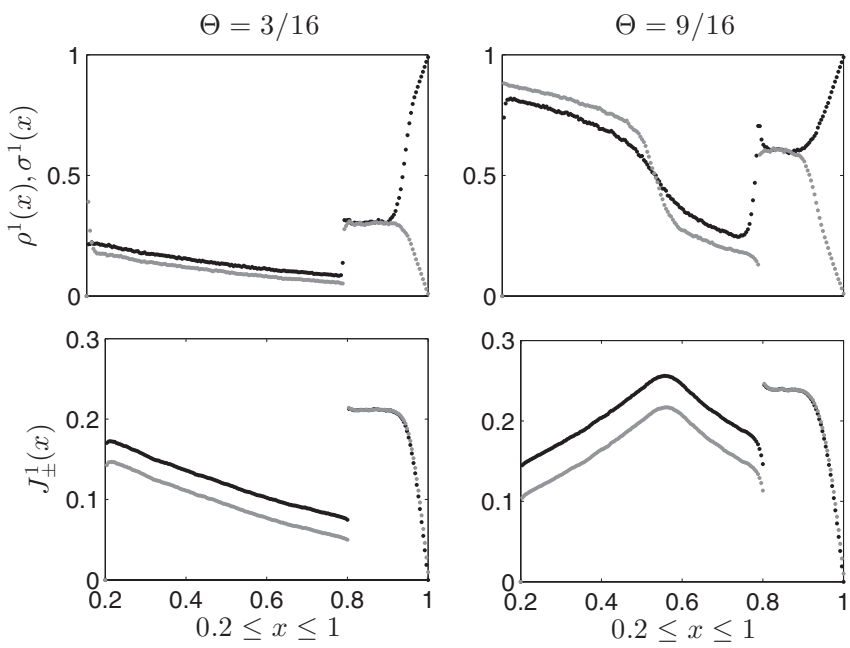

FIG. 12. Numerical current profiles (bottom) with corresponding density profiles (top) for overall densities $\Theta=3 / 16$ (left) and $\Theta=$ $9 / 16$ (right). Other parameters are $\Omega=5, q_{1}=0.8$, and $q_{2}=0.4$. Black dots are for plus-type particles, while gray dots are for the minus type. consider the average unidirectional current

$$
\left\langle J_{ \pm}\right\rangle_{x}:=\frac{\int_{0}^{1} J_{ \pm}(x) d x}{1-x_{1}}
$$

and define an overall current $\langle J\rangle_{x}:=\left\langle J_{+}\right\rangle_{x}+\left\langle J_{-}\right\rangle_{x}$. The dominance in current of particles can be investigated by looking at the ratio of average current for plus-type particles

$$
R_{+}:=\frac{\left\langle J_{+}\right\rangle_{x}}{\langle J\rangle_{x}}=\frac{\left\langle J_{+}\right\rangle_{x}}{\left\langle J_{+}\right\rangle_{x}+\left\langle J_{-}\right\rangle_{x}} .
$$

We similarly define the ratio of average currents within each section. In the unipolar sections, plus- and minus-type particles equally contribute to the average currents as the net current is zero. Thus the ratio in the entire bundle $R_{+}$depends only on the ratio within the bipolar section

$$
R_{+}^{b}:=\frac{\int_{x_{1}}^{x_{2}} J_{+}(x) d x}{\int_{x_{1}}^{x_{2}}\left[J_{+}(x)+J_{-}(x)\right] d x} .
$$

It is not difficult to see that when the density profiles in the bipolar section are in the LL phase $(\rho, \sigma \ll 1 / 2)$, dominance in current is equivalent to dominance in occupancy, $R_{+}^{b} \approx F_{+}^{b}$ (see Fig. 12, left panel); however, when shocks form in the bipolar section, higher densities (above one half) can give a lower current (see Fig. 12, right panel).

Dominance in occupancy does not imply dominance in current. More interestingly, by comparing the two panels in Fig. 11, $R_{+}^{b}$ shows a larger range than $F_{+}^{b}$ on changing parameters. Thus, for an intermediate overall density, it is important to consider the current rather than simply the occupancy when trying to determine which type of particles dominant the transport. In addition, we can see that the ratio in current $R_{+}^{b}$ shows a larger range on changing $q_{1}$ than on changing $q_{2}$ in this example. This suggests that $q_{1}$ is more important than $q_{2}$ for determining dominance in current.

\section{DISCUSSION}

In this paper we introduce an ASEP-type model to describe the bidirectional motility of particles on an oriented bundle of tracks. This aims to model the motion of cargo-motor complexes undergoing transport along an antipolar MT bundle within a cell. Our model is certainly a great simplification of cell transport processes. It is parametrized by the particle turning rate $\Omega$ (inversely proportion to the run length), the overall density $\Theta$ (the proportion of sites that are occupied by particles), and the obstacle- or end-induced switching rates $q_{1,2}$ (i.e., the rate at which particles switch MTs at the junctions between the unipolar and bipolar sections). We use numerical simulations and mean-field approximation to investigate the dependence of the stationary density profiles within the bundle on these parameters.

We observe that, as expected, the switching rates $q_{1,2}$ have a major effect on the distribution of particles along the bundle. Although we have not fully explored the dependence of phases on parameters, we highlight below a number of interesting features about the system. Even for low overall density $\Theta$, particles (cargo-motor complexes) can queue to form accumulations at minus ends in addition to any plus-end queuing observed in simpler situations [20]. The critical value 
of the end-induced switching rate $q_{2}$ above which shocks are formed at minus ends is investigated in Sec. III C. For intermediate overall density $\Theta$, we find a variety of phases, including smooth connection phases where the density profile of one type of particle on a track smoothly passes through one half, while the other type displays a shock in the limit $N \rightarrow \infty$. We discuss a variety of other phases and have investigated the role of the switching rates $q_{1,2}$ in determining which motor is dominant in the transport. For low overall densities, the obstacle-induced switching rate $q_{1}$ influences the fraction of particles of one type within the bipolar section in an approximately linear way. For high overall density $\Theta$, our study shows that although the fraction of occupancy by different type particles does not vary much with rates $q_{1,2}$, the particle type that actually contributes most to the current (i.e., transport) may vary much more.

Our model is inspired by in vivo experimental observations, although the model has been simplified in many ways as we now discuss. It is probable that hopping between MTs is not restricted to the ends of the bipolar section, but may occur throughout the bipolar section [21]. This would allow more possibilities for transition events and the possibility of plustype particles (in addition to minus-type particles) switching MTs. A switch in transport direction can result from hopping between MTs and the activity of kinesin-3 alone or it could be a consequence of dynein binding to the cargo, which in the case of EEs was shown to override kinesin-3 activity [19]. Our model also assumes only two MTs and only two lanes on each track, but it is known that numerous MTs form a bundle [21] and each MT consists of 13 protofilaments [17]. Thus many more tracks might support bidirectional motility of the cargo-motor complexes and allowing opposite-directed particles moving on the same protofilament will certainly give additional effects such as increasing the jamming at MT plus ends, as discussed in [16]. The complex geometry of the bundle may contribute to additional effects of cooperative transport, already considered for unidirectional transport in [29].

Notwithstanding these simplifications, we suggest the model is useful in a number of ways, especially when improved measurement of in vivo transition rates become possible, and we highlight some of these below.

(i) For low overall density of particles, it is possible to have an accumulation of particles at minus ends of MTs. As in vivo experiments so far show no obvious accumulation of early endosome cargos throughout the entire cell [21], if this model is accurate then it suggests that the switching rate $q_{2}$ must be relatively high in vivo. There are clearly other possible explanations due to features not included in the model such as a high turning rate of minus-directed organelles at minus ends (which might be due to an accumulation of motors at the minus end taking dynein to plus ends) can avoid such an accumulation of EEs; this mechanism would be a similar to that suggested in $[18,20]$, where an accumulation of dynein motors increases the turning rate of EEs near plus ends and so avoids accumulations of EEs.

(ii) For low overall density of cargos, the fraction of occupancy calculated in Sec. IV predicts how the proportion of EEs carried by kinesin-3 among all EEs (carried by either kinesin-3 or dynein) varies with the parameters. This fraction can in principle be explored experimentally to test the validity of the modeling assumptions for in vivo bidirectional transport.

The model discussed in this paper gives in some sense the simplest system that incorporates both bidirectional transport on a single MT and the antipolar bundling found in the living cell. As such, it has enabled us to investigate the interplay between the two possible causes of bidirectional transport: two populations of motors and the presence of antipolar arrangements of MTs.

\section{ACKNOWLEDGMENTS}

This work was supported by a grant from the Biotechnology and Biological Sciences Research Council BB/J009903/1.

\section{APPENDIX A: DENSITY PROFILES FOR NONZERO NET CURRENT}

In the bipolar section, the net current $J$ (as discussed in Sec. III) given by $J=\rho(1-\rho)-\sigma(1-\sigma)$ is constant when the system is in a statistically stationary state. This densitycurrent relation gives

$$
\sigma=\frac{1}{2} \pm \frac{2 \rho-1}{2}\left(\sqrt{\frac{4 J}{(2 \rho-1)^{2}}+1}-1\right) \pm \frac{2 \rho-1}{2},
$$

which implies that the nearly-equal- and nearlycomplementary-density solutions are

$$
\begin{aligned}
& \sigma=\rho+\frac{2 \rho-1}{2}\left(\sqrt{\frac{4 J}{(2 \rho-1)^{2}}+1}-1\right) \\
& \sigma=1-\rho-\frac{2 \rho-1}{2}\left(\sqrt{\frac{4 J}{(2 \rho-1)^{2}}+1}-1\right) .
\end{aligned}
$$

Thus the first order ODE (1) from the mean-field approximation reads

$$
0=\frac{d \rho}{d x}+\frac{\Omega}{2}\left( \pm \sqrt{\frac{4 J}{(2 \rho-1)^{2}}+1}-1\right),
$$

with a general solution of nearly equal or nearly complementary density

$$
\left[\left(1+\frac{4 J}{(2 \rho-1)^{2}}\right)^{3 / 2} \pm 1\right](2 \rho-1)^{3}=\mp 12 J \Omega x+C
$$

for any nonzero net current $J$ on taking options in " \pm " and “干”.

\section{APPENDIX B: MEAN-FIELD ANALYSIS FOR AN LL-SL PHASE}

When both types of particles in the bipolar section are in low densities, they are dominated by the injection rates $\alpha_{ \pm}^{b}$, which are approximated by

$$
\alpha_{+}^{b}=\bar{\sigma} q_{1}, \quad \alpha_{-}^{b}=\bar{\sigma}\left(1-q_{1}\right)
$$

where $\bar{\sigma}$ is the equal density in the unipolar section. Hence, from (5) and (6), the densities on the first track in the bipolar section are approximated by

$$
\rho=-J \Omega\left(x-x_{1}\right)+\alpha_{+}^{b}, \quad \sigma=-J \Omega\left(x-x_{2}\right)+\alpha_{-}^{b} .
$$


Together with the approximation $J=\rho-\sigma$, we find

$$
J=\frac{\alpha_{+}^{b}-\alpha_{-}^{b}}{\Omega\left(x_{2}-x_{1}\right)+1}=\frac{\bar{\sigma}\left(2 q_{1}-1\right)}{\Omega\left(x_{2}-x_{1}\right)+1},
$$

which gives a zero net current if $q_{1}=1 / 2$. The density expressions (B2) together with the symmetry $\rho^{1}(x)=\rho^{2}(1-$ $x)$ and $\sigma^{1}(x)=\sigma^{2}(1-x)$ give

$$
\rho^{1}(x)+\sigma^{2}(x)=\rho^{2}(x)+\sigma^{1}(x)=\bar{\sigma}, \quad x \in\left(x_{1}, x_{2}\right) .
$$

Thus

$$
\Theta=\frac{\int_{x_{1}}^{1} \rho(x)+\sigma(x) d x}{2\left(1-x_{1}\right)}=\frac{\bar{\sigma} / \Omega+(1-2 \bar{\sigma} / \Omega) \bar{\sigma}}{2\left(1-x_{1}\right)} .
$$

That is, given a low overall density $\Theta$, a sufficiently large $q_{2}$, and assuming densities are low in the bipolar section, we have

$$
\bar{\sigma}=\frac{1+\Omega}{4}-\sqrt{\frac{(1+\Omega)^{2}}{16}-\left(1-x_{1}\right) \Omega \Theta .}
$$

Substituting (B5), (B3), and (B1) into (B2) gives the analytical approximation of the density profiles in the bipolar section in an LL-SL phase. This, together with density profiles in the unipolar sections, gives the density profiles in the entire bundle.
[1] R. D. Vale, Cell 112, 467 (2003).

[2] S. P. Gross, Phys. Biol. 1, R1 (2004).

[3] K. Nishinari, Y. Okada, A. Schadschneider, and D. Chowdhury, Phys. Rev. Lett. 95, 118101 (2005).

[4] L. Hough, A. Schwabe, M. A. Glaser, J. R. McIntosh, and M. Betterton, Biophys J. 96, 3050 (2009).

[5] O. Campás et al., Biophys. J. 94, 5009 (2008).

[6] R. A. Blythe and M. R. Evans, J. Phys. A: Math. Theor. 40, R333 (2007).

[7] R. Lipowsky, S. Klumpp, and T. M. Nieuwenhuizen, Phys. Rev. Lett. 87, 108101 (2001).

[8] S. Klumpp and R. Lipowsky, J. Stat. Phys. 113, 233 (2003).

[9] M. Ebbinghaus and L. Santen, J. Stat. Mech. (2009) P03030.

[10] M. Ebbinghaus, C. Appert-Rolland, and L. Santen, Phys. Rev. E 82, 040901 (2010).

[11] M. Liu, K. Hawick, and S. Marsland, Phys. Lett. A 374, 516 (2010).

[12] S. Muhuri, L. Shagolsem, and M. Rao, Phys. Rev. E 84, 031921 (2011).

[13] M. R. Evans, D. P. Foster, C. Godrèche, and D. Mukamel, Phys. Rev. Lett. 74, 208 (1995).

[14] R. Juhász, Phys. Rev. E 76, 021117 (2007).

[15] T. Reichenbach, E. Frey, and T. Franosch, New J. Phys. 9, 159 (2007).

[16] C. Lin, G. Steinberg, and P. Ashwin, J. Stat. Mech. (2011) P09027.

[17] L. G. Tilney, J. Bryan, D. J. Bush, K. Fujiwara, M. S. Mooseker, D. B. Murphy, and D. H. Snyder, J. Cell Biol. 59, 267 (1973).

[18] M. Schuster, S. Kilaru, P. Ashwin, C. Lin, N. Severs, and G. Steinberg, EMBO J. 30, 652 (2011).

[19] M. Schuster, R. Lipowsky, M.-A. Assmann, P. Lenz, and G. Steinberg, Proc. Natl. Acad. Sci. USA 108, 3618 (2011).
[20] P. Ashwin, C. Lin, and G. Steinberg, Phys. Rev. E 82, 051907 (2010).

[21] M. Schuster, S. Kilaru, G. Fink, J. Collemare, Y. Roger, and G. Steinberg, Mol. Biol. Cell 22, 3645 (2011).

[22] J. H. Lenz, I. Schuchardt, A. Straube, and G. Steinberg, EMBO J. 25, 2275 (2006).

[23] R. Wedlich-Söldner, A. Straube, M. W. Friedrich, and G. Steinberg, EMBO J. 21, 2946 (2002).

[24] R. Wedlich-Söldner, M. Bölker, R. Kahmann, and G. Steinberg, EMBO J. 19, 1974 (2000).

[25] J. Brankov, N. Pesheva, and N. Bunzarova, Phys. Rev. E 69, 066128 (2004).

[26] E. Pronina and A. B. Kolomeisky, J. Stat. Mech. (2005) P07010.

[27] R. Wang, M. Liu, and R. Jiang, Phys. Rev. E 77, 051108 (2008).

[28] B. Embley, A. Parmeggiani, and N. Kern, Phys. Rev. E 80, 041128 (2009).

[29] I. Neri, N. Kern, and A. Parmeggiani, Phys. Rev. Lett. 107, 068702 (2011).

[30] M. J. I. Müller, S. Klumpp, and R. Lipowsky, Proc. Natl. Acad. Sci. USA 105, 4609 (2008).

[31] M. J. Müller, S. Klumpp, and R. Lipowsky, Biophys J. 98, 2610 (2010).

[32] A. G. Hendricks et al., Curr. Biol. 20, 697 (2010).

[33] V. Soppina, A. K. Rai, A. J. Ramaiya, P. Barak, and R. Mallik, Proc. Natl. Acad. Sci. USA 106, 19381 (2009).

[34] B. Derrida and M. R. Evans, J. Phys. A: Math. Gen. 32, 4833 (1999).

[35] D. T. Gillespie, J. Phys. Chem. 81, 2340 (1977).

[36] The unit $p$ is given by $p=v / h$, where $v$ is the velocity and $h$ is the space step, and the turning rate $\omega=v / M$. Hence the quantity $\Omega$ in unit of $p$ is given by $\Omega=\omega N / p=L / M$. 